\title{
Durable Benefit of Particle Occlusion of Systemic to Pulmonary Collaterals in Select Patients After Superior Cavopulmonary Connection
}

\author{
Michael L. O'Byrne' ${ }^{1,2} \cdot$ David N. Schidlow ${ }^{1}$
}

Received: 3 August 2017/ Accepted: 4 October 2017 / Published online: 7 October 2017

(C) Springer Science+Business Media, LLC 2017

\begin{abstract}
Systemic to pulmonary arterial collaterals (SPC) are commonly found in patients undergoing staged operative palliation for single ventricle heart disease. Occlusion of SPC as part of pre-Fontan catheterization has been shown to improve hemodynamics acutely. Anecdotally, the effect of this intervention appears to be transient, and to our knowledge there is no data supporting its durability in these patients. Between 1/1/2016 and 5/1/2017, 24 children underwent Glenn operations at our institution. Of these, 3 patients had signs and symptoms deteriorating clinical status suggestive of volume overload in the period between their Glenn operation and Fontan completion, prompting heart catheterization. SPC were occluded with a combination of polyvinyl alcohol embolization particles, and in some cases coils or vascular plugs. Clinical course and data from echocardiograms and serial catheterizations are presented. SPC occlusion was performed over 6 procedures in 3 subjects with technical success in each case. Hemodynamic evaluation was repeated in $2 / 3$ patients with improvement in collateral burden and hemodynamics in both cases. One patient previously thought to be unsuitable for Fontan completion improved sufficiently to undergo late Fontan completion, which was ultimately successful. In all patients, there was improvement in clinical status. In patients with severe SPC collateral durable benefit was seen, suggesting
\end{abstract}

Michael L. O’Byrne

obyrnem@email.chop.edu

1 Division of Cardiology, Children's National Medical Center and the George Washington University School of Health Sciences, Washington, DC, USA

2 Division of Cardiology and Center for Pediatric Clinical Effectiveness, The Children's Hospital of Philadelphia, 3401 Civic Center Blvd, Philadelphia, PA 19104, USA that in certain cases intervention on SPC remote from Fontan completion may have clinical benefit.

Keywords Pediatric cardiology · Hypoplastic left heart syndrome - Pulmonary atresia intact ventricular septum . Transcatheter intervention - Vascular embolization

\section{Introduction}

Collateral connections between systemic arteries and the pulmonary arterial bed are frequently seen in patients undergoing staged operative palliation of single ventricle heart disease. Over the course of staged palliation, SPC progressively become a less efficient contributor to pulmonary blood flow and an increasing volume load on the systemic ventricle. In early studies, occlusion of feeder vessels to SPC with coils had equivocal results on both hemodynamics and clinical outcomes [1-3]. Cardiac MRI has been used to quantify collateral burden [4-6] leading to an understanding that these vessels are highly prevalent $[4,5,7]$. The severity of SPC burden is variable. The mean collateral burden prior to Fontan is between 35 and 37\% of ascending aortic flow, but with some patients exceeding $50 \%$ of flow in the ascending aorta $[4,5,8]$.

Historically, transcatheter therapy for SPC was comprised of occluding proximal arteries feeding SPC with coils. More recently, injection of polyvinyl alcohol embolization particles has been adopted in the hopes that by reaching smaller caliber downstream vessels further downstream might reduce SPC burden more effectively. Occlusion of SPC with this technique has been shown to acutely improve cardiac output and effective pulmonary blood flow [9]. This has led some centers, including our own, to incorporate particle occlusion of SPC as part of 
routine pre-Fontan catheterizations in the hopes of improving post-operative outcomes.

However, less is understood regarding the hemodynamic consequences of SPC and their relationship to clinical status around the Fontan operation. Development of severe collateral burdens and the resultant ineffective pulmonary blood flow and systemic ventricular volume load has the potential to lead to progressive ventricular dilation, atrioventricular valve regurgitation (AVVR), and elevated end-diastolic pressure (EDP), along with a clinical syndrome of respiratory insufficiency, growth failure, and systemic venous hypertension. To our knowledge, there have not been previous reports of SPC implicated in precipitation of such a syndrome, nor have there been reports of transcatheter intervention to treat these symptoms.

In this case series, we detail the treatment of three patients with evidence of hemodynamic embarrassment attributable to SPC burden. In each case, attempts were made to systemically reduce collateral burden through transcatheter intervention. There was a high suspicion that SPC burden would recur, so patients were followed closely with plans for repeat catheterization to re-evaluate collateral burden. We observed that after one or more attempts treating SPC there was qualitative improvement in clinical status, echocardiograms, and hemodynamics on catheterization.

\section{Methods}

\section{Particle Occlusion of SPC}

In each case, occlusion of collaterals was accomplished in a similar fashion. A complete hemodynamic catheterization was performed quantifying evidence of hemodynamic derangement attributable to SPC, specifically: [1] step-up in oxygen saturation from the superior vena cava (SVC) to the pulmonary arteries (PA), [2] elevated systemic ventricular EDP and atrial pressures. Angiographic evaluation of SPC was also performed. On PA angiograms, wash-out of contrast by unopacified blood is seen in affected lung segments. SPC are more directly demonstrated in aortic arch angiograms with dilated feeder vessels of various sizes, opacification of the distal pulmonary arterial tree, and in severe cases backfilling of the branch PAs. We utilize the grading scheme originally described by Spicer et al. [1].

Next, a 4 Fr angled glide catheter (Terumo Interventional Systems, Somerset, NJ, USA) was advanced retrograde through an arterial sheath. Selective angiograms of each subclavian artery were performed, identifying branches from which feeder vessels to SPC originate. The catheter was then advanced distally and individual downstream branches were engaged sequentially starting distally and working proximally, engaging the lateral thoracic, thoracoacromial, and internal mammary arteries. In each vessel, an angiogram was performed, identifying whether they contributed significantly to the SPC burden. For arteries giving rise to significant SPC, the catheter was then advanced distally past any large branches, and a suspension of angiographic contrast and 700-900 $\mu \mathrm{m}$ Embosphere polyvinyl alcohol embolization particles (Merit Medical, South Jordan, UT USA) was injected. The catheter was then flushed with heparinized saline, followed by a hand injection angiogram through the same catheter to determine if collaterals were successfully occluded. This process was repeated as necessary to eliminate SPC from each feeder vessel.

Next, the intercostal arteries and true bronchial arteries were engaged, usually with the same angled glide catheter. In addition to the angled glide catheter, in certain cases, a 4 Fr Cobra catheter (Cardeon Corporation, Cupertino CA) more effectively engaged the intercostal vessels and was used to engage these vessels. Angiograms were performed, and remaining SPC were occluded as described previously. Attention was paid during initial angiograms to identify proximal branches of feeder vessels that had the potential to supply systemic tissue especially the spine (e.g. the anterior spinal artery off of the intercostal arteries), and care was taken to insure that particles were injected downstream of these branches. In some cases, this required advancing a Progreat microcatheter (Terumo Interventional Systems, Somerset, NJ, USA) co-axially through the end hole catheter to achieve distal position. A more dilute suspension of embolization particles and contrast was then injected directly through the microcatheter, followed by a similar routine of flushing the catheter with heparinized saline and post-intervention angiography.

The arteries feeding SPC are typically arteries that normally supply the chest wall and surrounding muscles form interdigitating connections which result in redundant supply to SPC (e.g. ipsilateral lateral thoracic and internal mammary arteries supply the same segments of the upper lobe) [10]. In some cases, we also noted larger caliber direct connections between these feeder vessels. To avoid inadvertently passing particles into other vascular beds, these large caliber connecting vessels were occluded before particles were injected, using either 0.038" MREye coils (Cook Medical, Bloomington, IN, USA) or an Amplatzer vascular plug 4 (St. Jude Medical, St. Paul, MN, USA). Otherwise, with one exception (case 3), because of suspicion that collaterals would recur, we did not attempt to occlude dilated proximal vessels. We hoped that this would allow us to access these feeder vessels if there was recurrence of collateral formation, rather than having to engage more obscure vessels. 
Because of the suspicion that collaterals would recur, each case was followed closely after catheterization with frequent clinic visits and transthoracic echocardiograms. Second or third catheterizations were performed in response to signs or symptoms of recurrent SPC.

\section{Results}

Between 1/1/2016 and 5/31/2017, 24 children underwent Glenn operations as part of staged single ventricle palliation at our institution. Of these, three cases underwent early transcatheter occlusion of SPC (Table 1).

Case 1 had pulmonary atresia with intact ventricular septum and a severely hypoplastic right ventricle (RV). Cardiac catheterization in the neonatal period demonstrated right ventricle-dependent coronary artery circulation (secondary to severe stenosis of the left main coronary artery). The patient experienced a bradycardic arrest and required pre-operative mechanical circulatory support with VA-ECMO before operative intervention with $3.0 \mathrm{~mm}$ right modified Blalock-Taussig shunt and removal of ECMO canulas. The post-operative course was notable for shunt stenosis requiring stent angioplasty with a $3.5 \mathrm{~mm} \times 15 \mathrm{~mm}$ Integrity coronary stent (Medtronic Medical, Fridley, MN, USA). Due to hemodynamic instability and later sternal wound infection, the sternum was left open for 24 days before eventual closure. After this protracted initial hospital course, patient was discharged home with close follow-up.

The patient underwent hemodynamic evaluation prior to a planned second-stage operation. The hemodynamics was auspicious, including an indexed pulmonary vascular resistance of $1.9 \mathrm{WU}$ per $\mathrm{m}^{2}$, and left ventricular (LV) EDP

Table 1 Description of cases

\begin{tabular}{|c|c|c|c|c|}
\hline Anatomic diagnosis & Preceding interventions & $\begin{array}{l}\text { Clinical } \\
\text { presentation }\end{array}$ & $\begin{array}{l}\text { SPC occlusion and other } \\
\text { interventions }\end{array}$ & $\begin{array}{l}\text { Post-intervention } \\
\text { course }\end{array}$ \\
\hline $\begin{array}{l}\text { 1. Pulmonary atresia intact } \\
\text { ventricular septum with right } \\
\text { ventricle-dependent coronary } \\
\text { circulation }\end{array}$ & $\begin{array}{l}\text { 1. Neonatal right modified BT } \\
\text { shunt. Pre-operative VA- } \\
\text { ECMO following a cardiac } \\
\text { arrest. Post-operative course } \\
\text { notable for prolonged open } \\
\text { chest and mediastinitis } \\
\text { 2. Bidirectional Glenn at } \\
4 \text { months of age }\end{array}$ & $\begin{array}{l}\text { Tachypnea and } \\
\text { increased work } \\
\text { of breathing } \\
\text { Persistent } \\
\text { diuretic } \\
\text { requirement } \\
\text { and admissions } \\
\text { with pleural } \\
\text { effusions }\end{array}$ & $\begin{array}{l}8 \text { months: particle } \\
\text { occlusion of SPC, } \\
\text { device occlusion of VV } \\
\text { collateral }\end{array}$ & $\begin{array}{l}\text { Resolution of } \\
\text { respiratory distress } \\
\text { Complete wean from } \\
\text { diuretics }\end{array}$ \\
\hline $\begin{array}{l}\text { 2. Heterotaxy syndrome, } \\
\text { unbalanced complete } \\
\text { common atrioventricular } \\
\text { canal (favoring the right), } \\
\text { double outlet right ventricle } \\
\{\text { A,D,D }\} \text { supracardiac total } \\
\text { anomalous pulmonary venous } \\
\text { return with subvalvar and } \\
\text { valvar pulmonary stenosis }\end{array}$ & $\begin{array}{l}\text { 1. Neonatal modified BT shunt } \\
\text { from left subclavian to LPA } \\
\text { 2. Bidirectional Glenn and } \\
\text { TAPVR repair at } 6 \text { months of } \\
\text { age }\end{array}$ & $\begin{array}{l}\text { Tachypnea and } \\
\text { increased work } \\
\text { of breathing } \\
\text { Persistent } \\
\text { diuretic } \\
\text { requirement } \\
\text { Growth failure } \\
\text { Severe AV valve } \\
\text { regurgitation, } \\
\text { severe } \\
\text { ventricular } \\
\text { dilation/ } \\
\text { dysfunction }\end{array}$ & $\begin{array}{l}13 \text { months: particle } \\
\text { occlusion of SPC coil } \\
\text { occlusion of the RIMA } \\
14 \text { months: particle } \\
\text { occlusion of SPC } \\
15 \text { months: particle } \\
\text { occlusion of SPC }\end{array}$ & $\begin{array}{l}\text { Complete resolution } \\
\text { of AVVR and } \\
\text { ventricular } \\
\text { dilation/ } \\
\text { dysfunction } \\
\text { Improvement in } \\
\text { respiratory distress } \\
\text { and symptoms } \\
\text { Removed from } \\
\text { transplant list due } \\
\text { to clinical } \\
\text { improvement }\end{array}$ \\
\hline $\begin{array}{l}\text { Pulmonary atresia intact } \\
\text { ventricular septum with } \\
\text { hypoplastic right ventricle }\end{array}$ & $\begin{array}{l}\text { 1. Neonatal PDA stent } \\
\text { 2. Bidirectional Glenn with clip } \\
\text { ligation of PDA stent at } \\
6 \text { months with near occlusion of } \\
\text { the LPA by the clipped stent. } \\
\text { LPA treated with balloon } \\
\text { angioplasty }\end{array}$ & $\begin{array}{l}\text { Poor growth } \\
\text { Symptoms } \\
\text { potentially } \\
\text { limited } \\
\text { because of } \\
\text { developmental } \\
\text { delay }\end{array}$ & $\begin{array}{l}39 \text { months: particle } \\
\text { occlusion of SPC, } \\
\text { occlusion of RIMA with } \\
\text { AVP4, and device } \\
\text { occlusion of VV } \\
\text { collateral } \\
42 \text { months: particle } \\
\text { occlusion of SPC, coil } \\
\text { occlusion of the RIMA } \\
\text { and LIMA }\end{array}$ & $\begin{array}{l}\text { Fontan completion } \\
\text { with } \\
\text { uncomplicated } \\
\text { post-operative } \\
\text { course and } \\
\text { discharge on } \\
\text { POD\#13 }\end{array}$ \\
\hline
\end{tabular}

$B T$ Blalock-Taussig, $D O L$ day of life, $P D A$ patent ductus arteriosus, $P O D$ post-operative day, $S P C$ systemic to pulmonary collaterals, $V A-E C M O$ venoarterial extra-corporeal membrane oxygenation, $V V$ veno-venous 
of $7 \mathrm{~mm} \mathrm{Hg}$. The patient underwent right bidirectional Glenn operation at 4 months of age. The post-operative period was notable for persistent tachypnea and respiratory effort requiring aggressive diuresis that was unable to be weaned during his hospitalization. He was discharged on POD 12.

As an outpatient, the patient had persistent tachypnea, respiratory insufficiency, and serous pleural effusions requiring multiple inpatient admissions and aggressive intravenous diuresis. Despite the pleural effusions, oxygen saturations remained persistently elevated in the low $90 \mathrm{~s}$. Transthoracic echocardiograms demonstrated diastolic flow reversal in the descending aorta consistent with significant runoff (Fig. 1) and, by color Doppler suggested the presence of relatively large caliber SPC. After discussion, the patient was referred for hemodynamic evaluation and possible intervention in the cardiac catheterization laboratory. The catheterization demonstrated a systemic arterial saturation of $81 \%$ with a mixed venous saturation of $52 \%$ in the superior vena cava (SVC), and step-ups to both pulmonary arteries (69\% in the right PA and $81 \%$ in the left PA). The saturation in the pulmonary veins was between 93 and $96 \%$. Though we acknowledged that in the presence of SPC there were heterogenous saturations in the distal PAs, no true mixed PA saturation, we concluded that the ratio of pulmonary to systemic blood flow was markedly elevated for a Glenn (and likely between 1.1 and 2.2 to 1). LV EDP had increased from pre-Glenn catheterization to $10 \mathrm{mmHg}$, and pressures throughout the Glenn circuit were markedly elevated $(20 \mathrm{mmHg})$. Angiography demonstrated severe (Spicer grade 4) SPC burden. An accessory hemiazygos vein that had been noted at his pre-Glenn catheterization was now markedly dilated and draining below the diaphragm. This veno-venous connection was addressed with an $8 \mathrm{~mm}$ Amplatzer Vascular Plug 24 (St. Jude Medical, St. Paul, MN, USA). Feeder vessels to SPC from the left lateral thoracic, thoracoacromial, and internal mammary arteries, the right lateral thoracic and internal mammary arteries, and several intercostal (right supreme and sixth and left third) arteries were occluded using the method described above. Recovery from catheterization was unremarkable and the patient was discharged.

During subsequent clinic visits, his work of breathing was noted to be markedly improved. He required no further admissions for intravenous diuresis, and was successfully weaned off of all diuretics over 8 months. The patient is currently 18 months old and is expected to undergo Fontan completion between 2 and 3 years of age after routine preFontan evaluation.

Case 2 had heterotaxy syndrome, unbalanced complete common atrioventricular canal (favoring the RV), double outlet RV $\{\mathrm{A}, \mathrm{D}, \mathrm{D}\}$ with supracardiac total anomalous pulmonary venous connection, and multilevel pulmonary

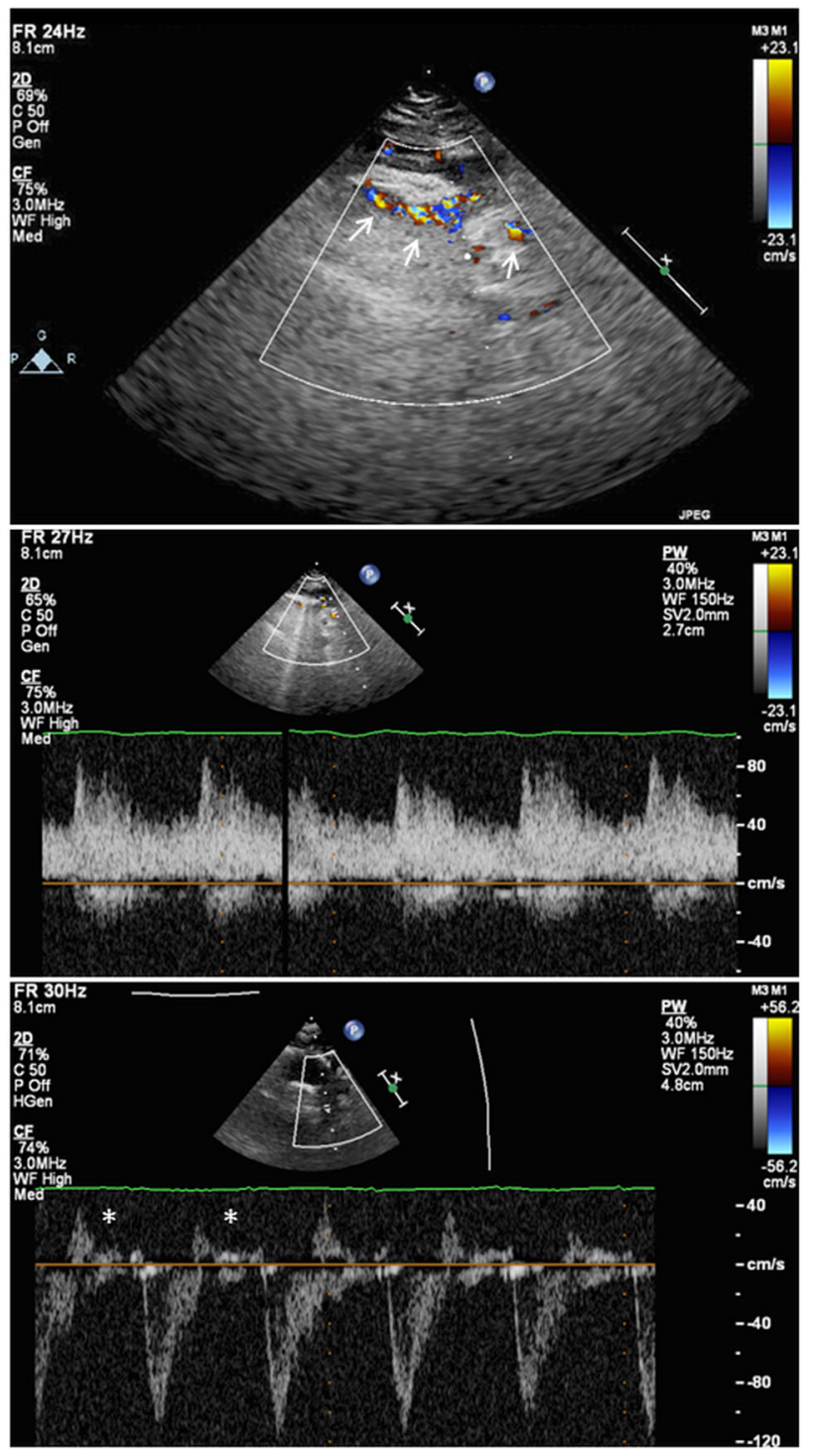

Fig. 1 Echocardiographic assessment of Case 1. Top Panel: Suprasternal color Doppler imaging demonstrates multiple signals (arrows) in the superior thorax consistent with systemic to pulmonary arterial collaterals. Middle panel: Spectral Doppler interrogation demonstrates a pulsatile pattern with continuous low-velocity flow in diastole consistent with the presence of systemic to pulmonary arterial collaterals Bottom panel: spectral Doppler sample of the proximal thoracic descending aorta demonstrating holodiastolic flow reversal (asterisk) consistent with a significant diastolic runoff due to systemic to pulmonary collateral burden

stenosis. The patient became progressively cyanotic, and a left modified Blalock-Taussig shunt was placed from the left subclavian artery to the LPA on DOL 16. Sternal closure was delayed and eventually performed on DOL 20. In the post-operative period, respiratory insufficiency and what appeared to be pulmonary over-circulation was noted. He received aggressive diuresis, and despite this was noted to have very poor growth, even with gastrostomy 
placement and tube feeds. The patient was discharged on DOL 78.

A pre-Glenn cardiac catheterization was performed at 4 months of age, demonstrating reasonable hemodynamics with a low indexed pulmonary vascular resistance (1.0 $\mathrm{WU} / \mathrm{m}^{2}$ ) in the face of a large magnitude left to right shunt (Qp:Qs of 3.5:1) and consequently high RV EDP $(14 \mathrm{mmHg})$. At 5 months of age, the patient underwent bidirectional Glenn and sutureless repair of his anomalous pulmonary veins and division of the anomalous connection to the SVC. Delayed sternal closure was performed on POD 2. He was discharged on POD 18. At discharge, an echocardiogram demonstrated unobstructed superior cavopulmonary and pulmonary venous anastomoses. There was mild AVVR and brisk RV systolic function. The patient was successfully weaned off of diuretics in the early post-operative outpatient period.

Over 6 months of outpatient follow-up, his status deteriorated considerably. Echocardiograms demonstrated progressive AVVR and deterioration of RV systolic
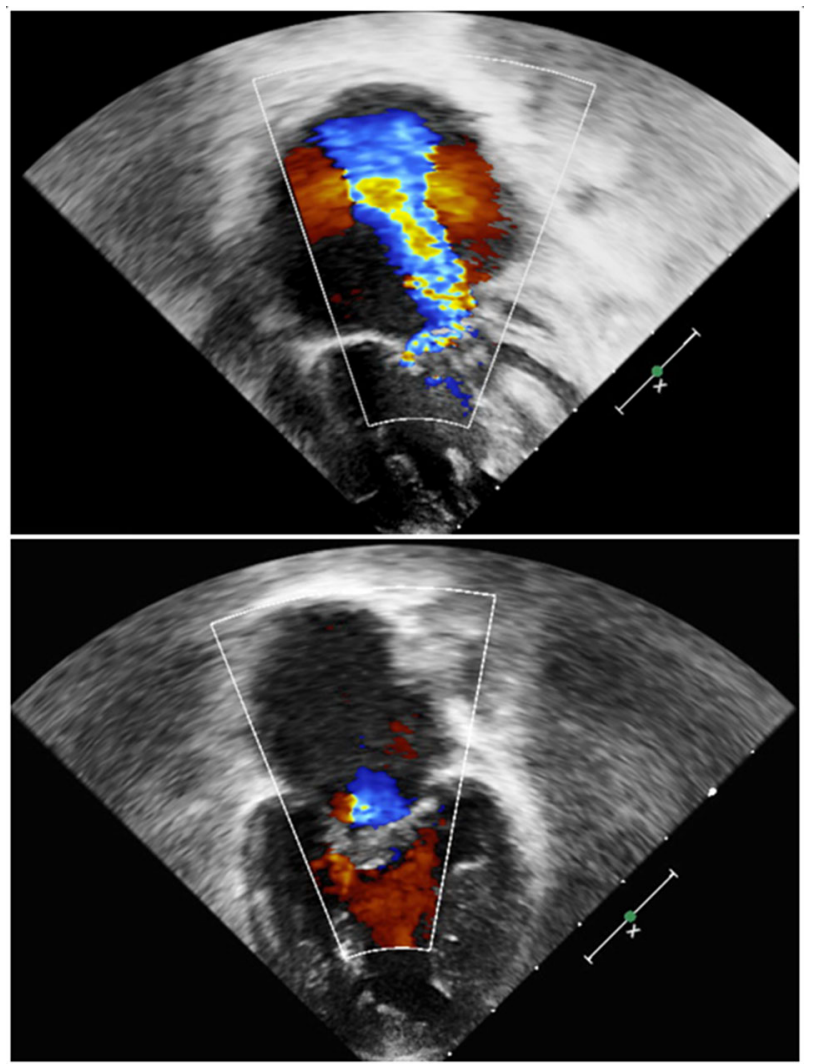

Fig. 2 Echocardiographic assessment of case 2. Top panel: 8 months after bidirectional Glenn there is severe atrioventricular valve insufficiency and severe common atrial enlargement. Bottom panel: After two intervening cardiac catheterization including occlusion of multiple systemic to pulmonary arterial collaterals and aggressive medical management, there is trivial residual atrioventricular valve insufficiency. The size of the common atrium is also significantly reduced function (Fig. 2). This was accompanied by progressive respiratory insufficiency, decreased growth velocity, and generalized edema. At 13 months of age, the patient was admitted for diuresis, respiratory support, and hemodynamic assessment. Similar to the first case, the systemic arterial saturation was generous at $83 \%$. There were significant step-ups from the SVC (48\%) to either PA (83\% on the right and $79 \%$ on the left) with saturations close to or reaching systemic arterial saturations. The pulmonary veins were fully saturated. There was a significant net left to right shunt (Qp:Qs between 2.3 and 3.2:1) along with elevated RV EDP (11 mmHg) and elevated Glenn pressures (mean of $19 \mathrm{mmHg}$ ). Angiography again demonstrated severe SPC burden (Spicer 4) (Fig. 3b). There was torrential collateral wash-out of contrast to the right lung such that contrast from a power injection in the SVC did not opacify the right PA at all (Fig. 3a). SPC off of the lateral thoracic and internal mammary arteries on each side as well as the fourth through ninth intercostal arteries on the right were occluded (Fig. 4). There was a relatively large caliber collateral vessel from the right internal mammary artery to the right PA with rapid transit of contrast. Before particles were injected this vessel was occluded with a series of three $0.038^{\prime \prime}$ MREye Coils. The patient underwent two subsequent cardiac catheterizations, with a further reduction in Qp:Qs to $1.3: 1$ and then $0.5-0.8: 1$. Concomitantly, the patient was initiated on an aggressive heart failure regimen, including a milrinone infusion. After recovering in the cardiac intensive care unit, echocardiograms demonstrated near complete resolution of the atrioventricular valve insufficiency (Fig. 2) and normalization of right ventricular systolic function. The patient was weaned to oral diuretics and an oral ACE inhibitor, which have been progressively weaned over a period of 8 months without change in RV function or AVVR. The patient was initially listed as status 2 for heart transplantation, but due to his marked improvement and stable status has now been delisted with a plan to ultimately pursue Fontan completion.

Case 3 also had pulmonary atresia and intact ventricular septum with severe RV hypoplasia, obligating a single ventricle palliation. The patient also has duodenal atresia, monosomy 7 , and partial trisomy 15 . The patient underwent stent angioplasty of the patent ductus arteriosus in the newborn period. He had a stable post-ductal stenting outpatient course. At 4 months of age, a pre-Glenn cardiac catheterization demonstrated a PVRi of $2.1 \mathrm{WU} / \mathrm{m}^{2}$, and he subsequently under bidirectional Glenn and clip ligation of the PDA stent.

The patient underwent balloon angioplasty of the left PA at 7 months of age because of severe LPA stenosis. At that catheterization, in addition to the aforementioned stenosis, severe collateral burden to the left lung was present, with 


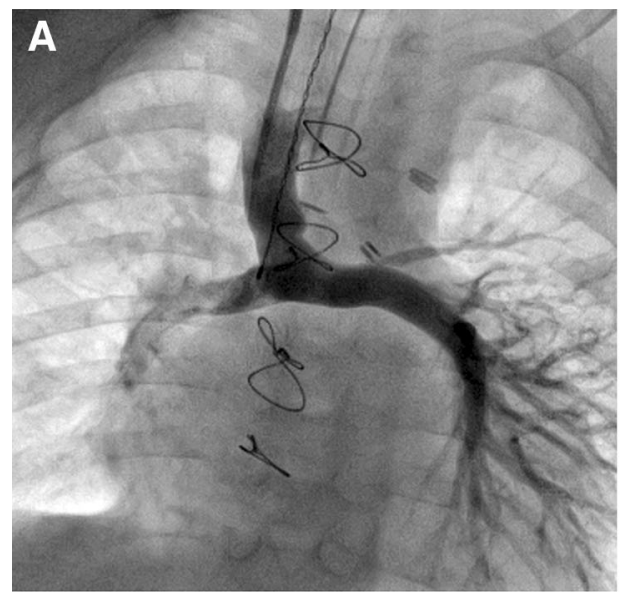

Fig. 3 Angiographic evaluation of collaterals. a Power injection angiogram (AP projection) through an antegrade Berman catheter whose sideholes are in the superior vena cava demonstrates a normal caliber superior cavopulmonary connection and branch pulmonary arteries. Severe wash-out of unopacified blood is seen in the right lung, such that contrast does not opacify the lobar branches of the right pulmonary artery. On the left side, there is normal opacification of the lower lobe. Severe wash-out is also seen in the left upper lobe. b Power injection angiogram (AP projection) through a retrograde pigtail catheter whose sideholes are positioned in the transverse aortic

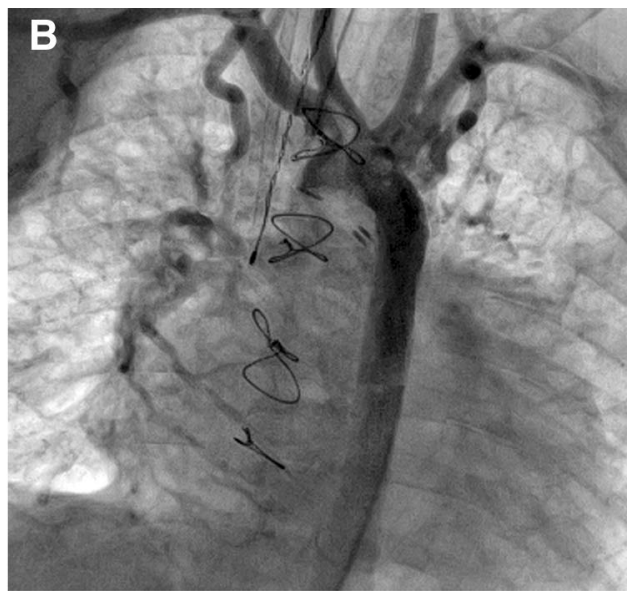

arch demonstrates severe systemic to pulmonary collateral burden. Both internal mammary arteries are dilated. The thoracoacromial arteries are also dilated, but less densely opacified because of run off into the internal mammary arteries. There is dense opacification of the right pulmonary artery in all three lobes with reflux of contrast to the right pulmonary artery proper. Contrast in the left lung is restricted to the upper lobe and backfilling of the left pulmonary artery is faint. This reflects severe systemic to artery collateral burden to the right lung with less burden in the left lung

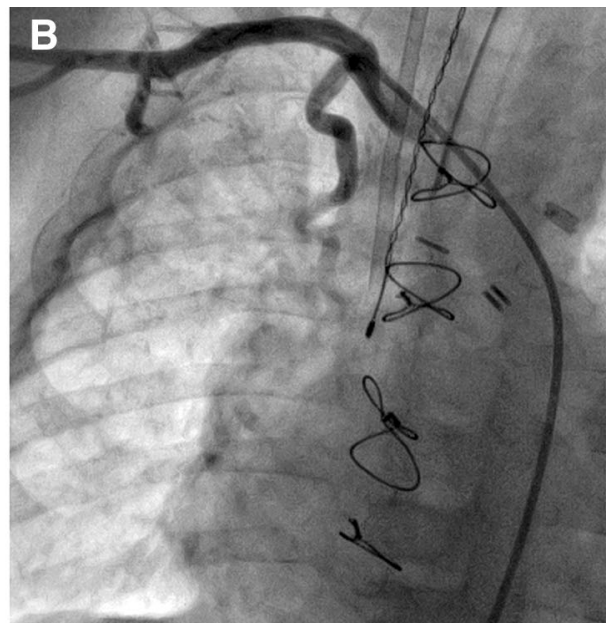

veins (not shown). b A similar angiogram performed after the thoracoacromial artery was engaged and embolization particles were injected into the vessel. The proximal dilated vessel remains intact, but contrast no longer passes downstream to the lung parenchyma. This demonstrated successful occlusion of the feeder vessels to the SPC in the right upper lobe

from the SVC (62\%) to both PA's (72\% in the right and $84 \%$ in the left) with fully saturated pulmonary veins (94-96\%) and a resultant Qp:Qs of 1.0-2.0:1. LV EDP was elevated $(14 \mathrm{mmHg})$ as were Glenn pressures (mean $25 \mathrm{mmHg}$ ). Angiography demonstrated torrential SPC burden with no contrast traversing the left lung on an SPC or left PA angiogram. The LV EDP and elevated Glenn 
circuit pressures suggested that the patient was not suitable candidate for Fontan completion. His medical regimen was adjusted, increasing the amount of diuretic received, and initiating enteral sildenafil.

Despite the inauspicious hemodynamics at the previous catheterization, the patient's family requested that an attempt be made to complete the Fontan. They consulted with the patient's cardiologist and surgeon, who requested that a repeat catheterization be performed and that any/all steps be taken to reduce the risk of Fontan completion. This was performed 11 months after the initial catheterization at 39 months of age. Hemodynamics was unchanged. In addition to conventional SPC, there were large caliber connections from the right internal mammary artery with rapid transit to the pulmonary arteries and veins on angiography. These connections were occluded with two Amplazter Vascular Plug 4 devices followed by embolization particles. SPC occlusion was performed for feeder vessels from both internal mammary arteries along with the right lateral thoracic and left thoracoacromial arteries. Following intervention, the Glenn pressure decreased to $20 \mathrm{mmHg}$ despite significant volume of intravenous fluids a contrast given over the course of a lengthy procedure.

As per routine, expeditious Fontan completion was scheduled. However, the operation was delayed for 3 months due to social reasons. At the request of the patient's surgeon, a repeat catheterization was performed to assess their suitability for Fontan completion. At that time, the Qp:Qs, LV EDP $(10 \mathrm{mmHg})$, and Glenn pressures (mean $17 \mathrm{mmHg}$ ) were all improved. There was reduced but still severe (Spicer 4) SPC burden, so particle occlusion of collaterals form the right thoracoacromial and internal mammary arteries and left lateral thoracic, thoracoacromial, thyrocervical trunk, and internal mammary arteries were performed. At the request of the patient's surgeon, both dilated internal mammary arteries were coil occluded with a total of fourteen $0.038^{\prime \prime}$ MREye coils of various diameters and lengths.

The patient underwent Fontan completion 5 days after catheterization. Post-operative course was notable for minimal bleeding noted in the operating room and rapid progress in the cardiac intensive care unit. Chest tubes for pleural effusions were removed on POD 9. He was discharged on POD 12 and has been well since discharge.

\section{Discussion}

In this case series, we describe three patients with Glenn circulations in whom severe SPC burden resulted in severe ventricular volume overload and clinical deterioration. In two cases, clinical deterioration induced us to pursue transcatheter intervention with little hope of durable results. In both cases, however, SPC occlusion resulted in a marked and durable improvement in clinical status and near complete resolution of associated volume overload. In the first case, this resulted in a significant improvement in respiratory status and diminished diuretic requirement. In the particularly dramatic example of the second case, severe AVVR and RV dysfunction resolved completely, permitting our group to no longer recommend heart transplantation and to resume pursuing staged palliation. In the third case, the patient had hemodynamics consistent with a high risk of Fontan failure, but after SPC occlusion they had marked improvements in hemodynamics and ultimately were able to undergo Fontan completion without complication. In three cases over 6 separate catheterization procedures, occlusion of the SPC was safe and effective.

The durable response of these patients to transcatheter intervention was surprising. At our institution, we routinely perform particle embolization of SPC as part of pre-Fontan catheterization. When there is evidence of significant volume overload (elevated EDP, step-up to either PA) or isolated angiographic evidence of significant collateral burden (Spicer grade $\geq 3$ ) collateral embolization is performed. Anecdotal experience has demonstrated that in this population there is a potential for recurrence over periods of weeks to months. Whether this is due to overlapping redundant connections between somatic arteries of the thorax or compensatory angiogenesis is not known. Because of this, we suspected that occlusion of SPC without subsequent Fontan completion would have only transient benefit. However, these cases suggest that under unusual circumstances, the volume load from SPC is sufficient to result in clinical deterioration and that the identification of these patients and treatment with SPC can effectively improve hemodynamics and reduce the need for medications and hospitalization.

In light of these results, it would be useful to identify patients at risk for profuse SPC burden and consider catheterization and intervention in those with respiratory insufficiency, poor somatic growth, atrioventricular valve regurgitation, and/or ventricular dysfunction. In addition, these extreme cases highlight the importance of primary prevention of SPC formation. At this time, the biological mechanisms for SPC formation are not well understood. Cyanosis and chest wall inflammation have been identified as two potential factors in this pathway. Several crosssectional studies have attempted to address by assessing association between collateral burden on MRI and suspected risk factors $[5,11,12]$. Supporting the notion that chest wall inflammation is associated with increased SPC burden, a history of modified Blalock-Taussig shunt along with prolonged hospitalization (quantified as intensive care time, chest tube time, ventilator time) at either the first and 
second-stage operations are associated with increased SPC burden in pre-Fontan patients [8, 13]. Studying the contribution of cyanosis has been challenging. History of hyper-cyanotic episodes were not associated with increased SPC burden [8], and in at least one study, patients with higher SPC burdens had higher oxygen saturation at discharge from their superior cavopulmonary connection hospitalization than those with lower SPC burden [13]. It is possible that this association is the result of higher early SPC burden as an augmenting source of Qp, rather than the cause of SPC. It is also possible that the cyanosis is confounded by the fact that several causes of cyanosis (e.g. effusion or mediastinitis) and interventions for cyanosis (re-opening or leaving open the sternum post-operatively) could potentially induce inflammation in the chest wall. At the same time, SPC are seen in patients critically decreased pulmonary blood flow [14] or pulmonary vein stenosis/ atresia [15] which also support the notion that SPC may be a compensatory process in the face of cyanosis, pulmonary blood flow, or both.

In terms of potentially modifiable practices potentially associated with SPC formation, delayed sternal closure is an interesting candidate. In previous studies, it was not associated with SPC burden, but in these series the practice was rare. Delayed sternal closure has been implicated as increasing the risk of post-operative wound infection and exposes the patient to a greater risk of inflammation in the post-operative period [16]. Also, with delayed sternal closure after the Glenn operation, patients are obligated to remain under obligate positive pressure ventilation which increases intrathoracic pressure, decreases pulmonary blood flow, and increases the potential for opening decompressing veno-venous collaterals all of which reduces patient oxygen saturation. In the current series, 2/3 cases had delayed sternal closure after stage 1 and 2 operations, and Case 1 had $>20$ days of open chest and a clinical diagnosis of mediastinitis. Cases 1 and 3 also had developed decompressing VV collaterals. All three cases also had periods of significant cyanosis with case three experiencing near chronic near occlusion of the left PA. Delayed sternal closure has a potentially important role in the care of the post-operative patient at the discretion of the operating surgeon. However, the findings of the current study may add to the accumulating literature that this practice should be reserved for high risk patients and that techniques (e.g. modified ultrafiltration) allowing for early chest closure may be beneficial to single ventricle patients.

We acknowledge that this case series is insufficient to make broad inferences about factors that predispose patients towards SPC formation. However, it highlights that in some patients, SPC formation can undermine hemodynamic stability and, importantly, this may be a process, which can be ameliorated or reversed. Future research is necessary to clarify whether there are modifiable practices to reduce the risk of SPC formation in terms of the operative approach and care of patients in between operations. At an individual patient level, cardiologists should consider SPC as a potentially treatable lesion in single ventricle patients with otherwise unexplained hemodynamic deterioration. As described above, previous series have described factors associated with the development of SPC, but identifying patients who might benefit from occlusion of SPC early after their Glenn depends more on clinical status and non-invasive imaging than any of these factors. In extreme cases, SPC are large enough to be seen during careful examination with color Doppler, and careful velocity map measurements by MRI can measure the degree of volume overload by measuring the percentage of cardiac output composed of SPC flow. However, less specific clinical (discrepantly high saturations along tachypnea, respiratory distress, and hepatomegaly) and echocardiographic (dilation of the systemic ventricle and progressive atrioventricular valve regurgitation) indicators may be more sensitive and identify patients in need of evaluation earlier in their course. Though the risks of a hemodynamic evaluation and possible intervention have to be balanced against the potential benefit to the patient and clinical status of the patient, our small experience suggests that in a subset of patients with SCPC a more aggressive approach may be of benefit in some patients. We concede that further research is necessary to determine the degree to which the benefits seen in this series are generalizable. It may be true that, in patients with more moderate SPC burden, early intervention has less net benefit.

As described above, catheterization with assessment of collaterals has been a standard component of the preFontan evaluation at our institution in patients with evidence of large magnitude SPC burden. Though not described in this series, there are also a subset of postoperative Fontan patients with prolonged pleural drainage who have undergone catheterization and undergone particle occlusion of SPC. This has been followed by rapid resolution of pleural effusions, suggesting that the collaterals are part of the pathophysiology of these effusions. There is limited experience with this procedure in Fontan patients beyond the perioperative period. We have not encountered patients with torrential SPC burden with or without associated symptoms of heart failure late after Fontan completion, and, to our knowledge, this has not been reported elsewhere. Ongoing research about (1) the unnatural history of collateral burden through staged palliation and (2) the biological mechanisms underlying the development of these pathological connection may clarify this. 


\section{Conclusion}

While acknowledging these limitations, this case series highlights that SPC burden can result in hemodynamic embarrassment in patients following Glenn operations, and that transcatheter intervention can have durable benefits, even remote from Fontan completion. Consideration should be given to catheterization and possible intervention in cases with otherwise unexplained volume overload, ventricular dysfunction, and/or AVVR in patients with single ventricle heart disease.

Funding Dr. O'Byrne receives research support from the National Institute of Health/National Heart Lung and Blood Institute (K23 HL130420-01). The funding agencies had no role in the drafting of the manuscript or influencing its content. This manuscript represents the opinion of the authors alone.

\section{Compliance with Ethical Standards}

Conflicts of interest The authors have no financial conflicts of interest to disclose.

\section{References}

1. Spicer RL, Uzark KC, Moore JW, Mainwaring RD, Lamberti JJ (1996) Aortopulmonary collateral vessels and prolonged pleural effusions after modified Fontan procedures. Am Heart $\mathbf{J}$ 131(6):1164-1168

2. McElhinney DB, Reddy VM, Tworetzky W, Petrossian E, Hanley FL, Moore P (2000) Incidence and implications of systemic to pulmonary collaterals after bidirectional cavopulmonary anastomosis. Ann Thoracic Surg 69(4):1222-1228

3. Banka P, Sleeper LA, Atz AM, Cowley CG, Gallagher D, Gillespie MJ et al (2011) Practice variability and outcomes of coil embolization of aortopulmonary collaterals before Fontan completion: a report from the Pediatric Heart Network Fontan CrossSectional Study. Am Heart J 162(1):125-130

4. Whitehead KK, Gillespie MJ, Harris MA, Fogel MA, Rome JJ (2009) Noninvasive quantification of systemic-to-pulmonary collateral flow: a major source of inefficiency in patients with superior cavopulmonary connections. Circ Cardiovasc Imaging 2(5):405-411

5. Grosse-Wortmann L, Al-Otay A, Yoo S-J (2009) Aortopulmonary collaterals after bidirectional cavopulmonary connection or Fontan completion: quantification with MRI. Circ Cardiovasc Imaging 2(3):219-225

6. Downing TE, Whitehead KK, Dori Y, Gillespie MJ, Harris MA, Fogel MA et al (2013) Accuracy of conventional oximetry for flow estimation in patients with superior cavopulmonary connection: a comparison with phase-contrast cardiac MRI. Circ Cardiovasc Imaging 6(6):943-949

7. Prakash A, Rathod RH, Powell AJ, McElhinney DB, Banka P, Geva T (2012) Relation of systemic-to-pulmonary artery collateral flow in single ventricle physiology to palliative stage and clinical status. Am J Cardiol 109(7):1038-1045

8. Prakash A, Satiroglu E, Porras D, McElhinney DB, Keane JF, Lock JE et al (2013) Risk factors for profuse systemic-to-pulmonary artery collateral burden in hypoplastic left heart syndrome. Am J Cardiol 112(3):400-404

9. Dori Y, Glatz AC, Hanna BD, Gillespie MJ, Harris MA, Keller MS et al (2013) Acute effects of embolizing systemic-to-pulmonary arterial collaterals on blood flow in patients with superior cavopulmonary connections: a pilot study. Circ Cardiovasc Interv 6(1):101-106

10. Stern HJ (2009) The argument for aggressive coiling of aortopulmonary collaterals in single ventricle patients. Catheter Cardiovasc Interv 74(6):897-900

11. Grosse-Wortmann L, Drolet C, Dragulescu A, Kotani Y, Chaturvedi R, Lee K-J et al (2012) Aortopulmonary collateral flow volume affects early postoperative outcome after Fontan completion: a multimodality study. J Thorac Cardiovasc Surg 144(6):1329-1336

12. Prakash A, Rathod RH, Powell AJ, McElhinney DB, Banka P, Geva T (2012) Relation of systemic-to-pulmonary artery collateral flow in single ventricle physiology to palliative stage and clinical status. Am J Cardiol 109(7):1038-1045

13. Glatz AC, Harrison N, Small AJ, Dori Y, Gillespie MJ, Harris MA et al (2015) Factors associated with systemic to pulmonary arterial collateral flow in single ventricle patients with superior cavopulmonary connections. Heart 101(22):1813-1818

14. Haroutunian LM, Neill CA (1972) Pulmonary complications of congenital heart disease: hemoptysis. Am Heart J 84(4):540-549

15. Grosse-Wortmann L, Hamilton R, Yoo S-J (2007) Massive systemic-to-pulmonary collateral arteries in the setting of a cavopulmonary shunt and pulmonary venous stenosis. Cardiol Young 17(5):548-550

16. Nelson-McMillan K, Hornik CP, He X, Vricella LA, Jacobs JP, Hill KD et al (2016) Delayed sternal closure in infant heart surgery-the importance of where and when: an analysis of the STS congenital heart surgery database. Ann Thoracic Surg 102(5):1565-1572 